\title{
Tumor primário de pulmão simulando doença de Alzheimer: relato de caso
}

\author{
Primary lung tumor simulating Alzheimer's disease: case report \\ José Eduardo Martinelli', Juliana Francisca Cecato ${ }^{1}$
}

\section{RESUMO}

Retratamos o relato de um homem de 61 anos que apresentava um glioblastoma multiforme em região do giro têmporo-occipital lateral esquerdo que rechaça cranialmente o hipocampo e o corno temporal do ventrículo lateral correspondente. Não havia sinais e sintomas neurológicos e o quadro clínico lembrava a demência de Alzheimer. Havia comprometimento de memória e alterações comportamentais com duração de três meses. Foram afastadas inicialmente outras causas de demência rapidamente progressivas e a ressonância magnética revelou a etiologia tumoral dos sintomas neuropsiquiátricos. Foi submetido à bateria de testes neuropsicométricos com pontuação compatível com doen-

\section{Palavras-chave}

Idoso, neoplasias encefálicas, diagnóstico diferencial, tratamento.

\section{Keywords}

Elderly, brain neoplasms, differential diagnosis, treatment. ça de Alzheimer (DA). Em razão de extensa área de edema, foi introduzida dexametasona com reversão completa dos sintomas neuropsiquiátricos iniciais e a repetição da bateria neuropsicológica mostrou melhora acentuada em todos os testes. Optou-se por tratamento conservador, após biópsia por agulha, com evolução para óbito em cinco meses.

\section{ABSTRACT}

We report a 61-year-old man case who presented a multiform glioblastoma located at the left side of the temporal-occiptal gyrus that reached the hippocampus cranially and the temporal horn of the left lateral ventriculus. There were no neurological signs and symptoms and the clinical picture reminded the Alzheimer's dementia. Memory impairment and behavioral alterations were observed during three months. Other causes of rapid progressive dementia were initially discarded and the magnetic resonance revealed a tumoral etiology for the neuropsychiatric symptoms. A battery of neuropsychometric tests were applied, with a score similar to AD. Due to the extensive edema's area, dexamethasone was introduced with complete reversal of the neuropsychiatric initial symptoms and the repetition of the neuropsychological battery showed a noticeable improvement in all the tests. A conervative treatment was chosen, after needle biopsy, evolving to death in five months.
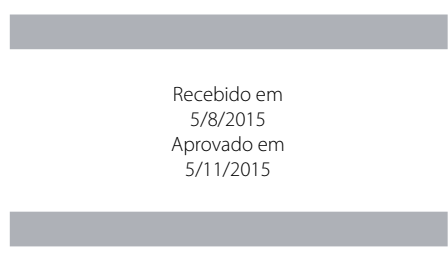

DOI: 10.1590/0047-2085000000111

\author{
Endereço para correspondência: José Eduardo Martinelli \\ Rua Prudente de Moraes, 111, VI Argos \\ 13201-004 - Jundiaí, SP, Brasil \\ E-mail: drmartinelli@terra.com.br
}




\section{INTRODUÇÃO}

Alterações mentais e cognitivas são observadas em 15\% a $20 \%$ dos tumores cerebrais e podem ser suas primeiras manifestações. A gravidade dos distúrbios cognitivos varia de transtornos de atenção e julgamento a síndromes maiores, tais como delirium, síndrome amnésica ou demência. Os problemas mentais variam de apatia, irritabilidade a depressão maior ou sintomas psicóticos ${ }^{1}$.

Os sintomas mentais ou cognitivos estão relacionados a muitos fatores, incluindo as características da lesão (natureza do tumor, localização, distribuição e rapidez de instalação), do paciente (constituição biológica, personalidade, experiência sócio-ocupacional e cultural) e ao ambiente psicossocial atual ${ }^{2}$. Os sinais e sintomas relacionados a neoplasias do sistema nervoso central (SNC) acontecem em consequência da invasão ou compressão do tecido cerebral pelo tumor. Tumores de crescimento lento (meningiomas, gliomas de baixo grau) geralmente apresentam grande volume quando diagnosticados devido à acomodação do tecido cerebral dentro da caixa craniana em determinado período de tempo. Tumores de crescimento rápido e que desencadeiam edema cerebral, por outro lado, podem desenvolver achados clínicos com pequeno volume tumora ${ }^{3-5}$.

Os idosos são um grupo especial, quando acometidos por tumores cerebrais, em virtude das alterações fisiológicas do envelhecimento cerebral e sua atrofia. Consequentemente, um aumento na pressão intracraniana e cefaleia não são observados nos estágios iniciais do desenvolvimento da doença, por essa razão o aparecimento de outros sintomas mentais inicialmente abrindo o quadro clínico'.

Na prática clínica diária, chama a atenção para a etiologia orgânica a presença de alterações da consciência, memória, atenção e concentração, predomínio de alucinações visuais, crises epiléticas e algum sintoma ou sinal focal lateralizado (heminegligência, afasia, agnosia, apraxia). As causas somáticas podem ser divididas em dois grandes grupos: um de localização intracerebral (traumas, tumores, hemorragias, tromboses, hidrocefalia e doenças degenerativas) e outro extracerebral (infecções sistêmicas, endocrinopatias, doenças toxicometabólicas). Dependendo das manifestações clínicas dos tumores (sinais neurológicos focais, convulsões ou consequentes do aumento da pressão intracraniana), os profissionais mais procurados são os neurologistas e neurocirurgiões. Os psiquiatras e geriatras serão procurados quando os sintomas iniciais forem mentais e psiquiátricos ou dependendo da idade do paciente?.

Num trabalho de Silva et al. ${ }^{8}$ em que foi realizada a investigação neuropsicológica pré-operatória em pacientes com glioma de baixo grau, todos os 13 pacientes investigados apresentaram déficits cognitivos, sendo a memória episódica verbal e as funções executivas as mais prejudicadas. Oito pacientes (62\%) apresentaram déficit grave de memória epi- sódica verbal e imediata, sete (54\%) de tardia e quatro (31\%) de reconhecimento. Quanto às funções executivas, $92 \%$ dos pacientes apresentaram déficit de fluência verbal nominal e 77\% de fluência verbal categórica, variando entre leve e grave. Todos tinham avaliação neurológica normal. A faixa etária variou de 25 a 60 anos. Talvez, se o grupo apresentasse uma faixa etária maior, os resultados encontrados seriam diferentes devido às alterações próprias do envelhecimento.

As alterações provocadas pelo tumor comprometem qualquer aspecto da função psicológica. Algumas vezes, determinadas áreas são afetadas isoladamente, como, por exemplo, o nível de consciência, mas pode haver acometimento de aspectos da função cognitiva ou estado afetivo, embora frequentemente várias áreas sejam afetadas concomitantemente 9 . Os distúrbios da função cognitiva são os mais frequentemente encontrados, como diminuição da capacidade de atenção e concentração, dificuldade de memória e fadiga mental. Um prejuízo mais grave da cognição pode se manifestar na forma de demência, com dificuldade de memória, diminuição da velocidade de processamento da informação e alteração das funções executivas (julgamento, abstração, sequenciação) ${ }^{10}$.

A incidência de tumores cerebrais nos pacientes com diagnóstico inicial de doença psiquiátrica é de 1\% a 2\% significativamente maior que na população. Os tumores supratentoriais, principalmente frontais e temporais, causam alterações cognitivas e comportamentais. Tumores de crescimento rápido podem apresentar quadros psicóticos, enquanto os de crescimento lento se manifestam com sintomas de alteração da personalidade, depressão ou apatia. Entre os principais tipos de tumores cerebrais, encontram-se os gliomas. Sua incidência é de 5 a 7: 100.00 habitantes ou 29\% de todas as neoplasias cerebrais. Eles se dividem em baixo e alto grau de acordo com seu aspecto histológico, variando de grau I (menos maligno) a grau IV (mais maligno) como o glioblastoma multiforme (GBM) ${ }^{7}$. A Organização Mundial da Saúde (OMS) usa um sistema trifásico de classificação em que o grau do tumor reflete a extensão de anormalidade morfológica das células tumorais (anaplasia), sua taxa aparente de crescimento e a presença de necrose. Os GBM são caracterizados por altas taxas de crescimento (mais de 20\%) e necrose. O grau do tumor é o melhor preditor do prognóstico. A biópsia por agulha está muitas vezes indicada principalmente em tumores que não podem ser removidos com segurança ${ }^{7}$.

A incidência de tumores cerebrais varia com a idade, com seu auge entre 65 a 70 anos. A incidência do glioblastoma é máxima aos 60 anos de idade. Quanto à localização, são mais comuns nos lobos frontal e temporal, podendo envolver mais de um lobo e dar implantes secundários?.

A maioria das demências na prática geriátrica pode ser considerada classicamente como crônica e evolui caracteristicamente de modo lento, no decurso de muitos anos. 
Entretanto, há um grupo de condições, as demências rapidamente progressivas (DRPs), que podem evoluir de modo subagudo em período menor que um ano, ou até em meses, semanas ou mesmo dias, podendo ser rapidamente fatais?. Muitas das DRPs são passíveis de tratamento e reversão. Portanto, a avaliação e o diagnóstico rápidos são importantes, já que pequenos períodos de tempo sem tratamento apropriado podem levar a sequelas irreversíveis.

As DRPs incluem condições que podem ser classificadas em neurodegenerativas, imunomediadas, vasculares, infecciosas, metabólicas, tóxicas, tumorais e psicogênicas. Podem ser encontradas no grupo etário senil e pré-senil, incluindo sua faixa mais jovem 5 . Entre as neurodegenerativas, a doença de Creutzfeldt-Jakob, a principal do grupo priônico, é a mais caracterizada das DRPs. As neurodegenerativas clássicas, porém, com evolução atípica.

A maioria das infecções do SNC que causam alteração do estado mental se manifesta de forma aguda. Porém, algumas se instalam de modo mais lento. Devemos considerar vírus, bactérias, fungos e parasitas. As infecções do SNC são acompanhadas por manifestações sistêmicas típicas de infecção, como febre e leucocitose ${ }^{11,12}$. As encefalites virais, geralmente agudas, são algumas vezes insidiosas. Os vírus herpes simplex (VHS-1 e VHS-2), Epstein-Barr, citomegalovírus, vírus varicela-zóster e enterovírus podem se manifestar sob a forma de alterações mais graduais do estado mental e do comportamento.

A encefalite pelo VHS-1 é a mais comum no adulto, com altas taxas de morbidade e mortalidade; deve ser sempre considerada na avaliação de alterações cognitivas e comportamentais de ação rápida.

Os critérios para demência do DSM-V ${ }^{13}$ foram substancialmente modificados em relação à versão anterior. Por ser uma classificação de doença, não inclui os estados pré-clínicos, mas acrescenta um estágio patológico pré-demência semelhante ao declínio cognitivo leve de Petersen et al. ${ }^{14}$. Introduz-se o conceito de transtorno neurocognitivo que ocupa o lugar dos transtornos mentais orgânicos. Desse modo, os transtornos neurocognitivos se dividem em três categorias: delirium, transtorno neurocognitivo menor e transtorno neurocognitivo maior. Os transtornos neurocognitivos maior e menor se diferenciam em função da intensidade dos sintomas e sua repercussão na funcionalidade do paciente. Os domínios sintomáticos estudados para o diagnóstico serão: atenção, função executiva, aprendizagem, memória, linguagem, funções visuoperceptivas, visuoconstrucionais e cognição social. O DSM-V inclui diferentes subtipos etiológicos dentro de sua classificação de transtornos neurocognitivos, entre eles o transtorno neurocognitivo devido à enfermidade de Alzheimer ${ }^{15}$.

O grupo de Dubois estabeleceu recentemente critérios diagnósticos para doença de Alzheimer (DA), não sendo de aplicação para outro tipo de demência. Para os autores, o fato principal do ponto de vista clínico é o comprometimento gradual e progressivo da memória episódica isolada ou associada a outras alterações cognitivas, mas sempre objetivada mediante os resultados dos testes neuropsicológicos. Os critérios de exclusão para esse grupo incluem a rapidez de instalação do quadro, a presença de enfermidades psiquiátricas ou de condições médica ou farmacológicas que possam justificar a clínica ${ }^{14,15}$.

Madhusoodanan et al.' recomendam que exames de neuroimagem sejam considerados nas seguintes condições: psicoses de início recente, alterações do humor e sintomas de perda de memória de início recente, ocorrência de novos sintomas ou sintomas atípicos, alterações de personalidade de início recente e anorexia sem sintomas corporais dismórficos.

\section{RELATO DE CASO}

Paciente SRP, 61 anos, sexo masculino, escolaridade de 8 anos, comerciante, procurou o ambulatório de geriatria. A acompanhante informou que, nos últimos três meses, seu pai vinha apresentando déficit progressivo de memória, como dificuldade em passar recados e em nomear objetos. Esquecia o combinado, perdia a sequência da conversa e estava repetitivo nas perguntas. Ao escovar os dentes, deixava a escova suja na sala, usava qualquer escova, deixava de limpar a lâmina de barbear, deixava de comprar itens da lista do supermercado, de pagar contas etc. Sempre gostou de fazer reparos domésticos, mas estava apresentando dificuldades.

O paciente não sabia referir os fatos contados pela acompanhante, mas reconhecia que estava esquecido. Não tinha queixa de cefaleia e tontura. Trabalhava bastante e estava há 15 anos sem férias, atribuindo esses problemas de esquecimento e confusão à estafa mental por excesso de trabalho. Não dormia bem à noite e, quando acordava, relatava dificuldade de adormecer novamente. A acompanhante atribuiu o aparecimento dos sintomas ao estresse financeiro intenso que passou há 90 dias, quando coincidiu o casamento de um filho e a formatura da filha (medicina).

Era hipertenso e gotoso há 10 anos. Usava indapamida 1,5 mg, olmesartana $40 \mathrm{mg}$ e alopurinol $300 \mathrm{mg}$ diariamente. A hipertensão e a artrite gotosa estavam compensadas com a medicação e não havia queixas relacionadas a essas doenças. Não apresentava antecedentes pessoais psiquiátricos e a história familiar é negativa para transtornos psiquiátricos maiores. Fumou dos 20 aos 30 anos de idade.

O exame físico mostrava pressão arterial de 120 por 80 $\mathrm{mmHg}$, frequência cardíaca $=76 \mathrm{bpm}$ e o paciente estava eupneico, corado e sem outros achados aos demais apareIhos. O exame neurológico não apresentava sinais localizatórios e seu fundo de olho era normal. Com suspeita inicial de síndrome demencial (DA), foi submetido à avaliação neuropsicológica por meio de: Miniexame do Estado Mental 
(MEEM) ${ }^{16}$, Cambridge Examination for Mental Disorders of the Elderly (CAMDEX) e sua bateria cognitiva (CAMCOG) ${ }^{17}$, Questionário de Atividades Funcionais de Pfeffer (QAFP) ${ }^{18}$, Teste do Desenho do Relógio ${ }^{19,20}$ e Escala de Depressão Geriátri$\mathrm{ca}^{21}$, no dia da primeira consulta. Foram realizados exames laboratoriais que são rotina da investigação diagnóstica na suspeita de DA e outros exames bioquímicos (THS, VDRL, Na, K, dosagem de vitamina B12, glicemia, ureia, creatinina, ácido úrico, TGO, TGP, GGT e hemograma), assim como a ressonância magnética do encéfalo.

Os exames laboratoriais estavam normais e a ressonância magnética (Figura 1) mostrou imagem nodular sólida ocupando o giro têmporo-occipital lateral esquerdo que rechaça cranialmente o hipocampo e o corno temporal do ventrículo lateral correspondente, que se encontra parcialmente obliterado. Apresenta-se com alto sinal em T2 e em Flair, com focos de "flow void" no seu interior. A restrição de difusão é variada e heterogênea. Há extensa área de edema perilesional.

Após o diagnóstico, foi introduzida dexametasona $12 \mathrm{mg}$ por dia, por via oral, e o paciente encaminhado à equipe de neurocirurgia. Por causa da grande melhora dos sintomas cognitivos após a corticoterapia, optou-se por fazer uma biópsia por agulha, que foi realizada 15 dias após o encaminhamento. $\mathrm{O}$ anatomopatológico revelou glioblastoma multiforme e do sistema nervoso central com necrose focal e extensas áreas gemistocíticas.

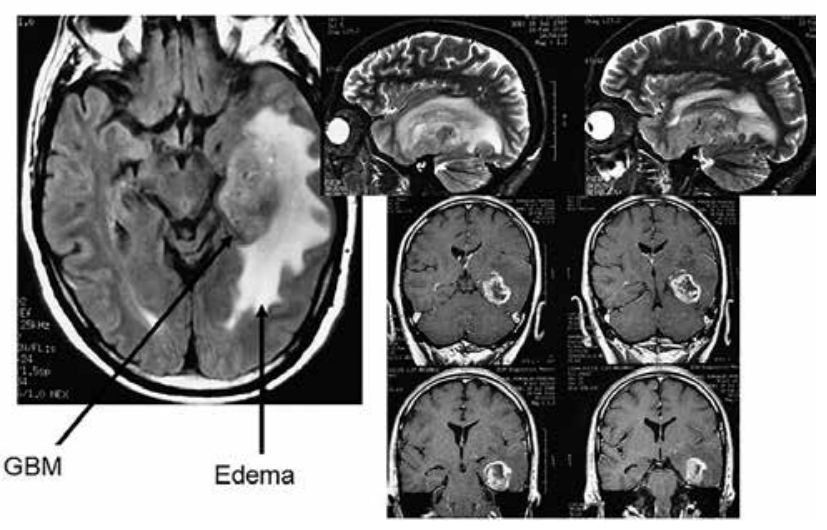

Figura 1. Ressonância magnética de crânio evidenciando tumor cerebral em região têmporo-occipital lateral esquerda.

\section{EVOLUÇÃo}

Com uma semana após o uso do corticoide, o paciente apresentou reversão quase completa dos sintomas cognitivos, a ponto de passar um fim de semana em uma pescaria com os amigos. Em função dessa melhora, foi realizada a mesma bateria dos testes neuropsicométricos, cujos resultados estão na Tabela 1.
Tabela 1. Escores esperados e a pontuação obtida pelo paciente na segunda avaliação neuropsicométrica, após administração de exametasona

\begin{tabular}{lcc}
\hline Testes & Resultados antes da cirurgia & Resultados pós-corticoterapia \\
\hline MEEM & 22 & 28 \\
CAMCOG & 68 & 82 \\
Mendez & 17 & 19 \\
Shulman & 3 & 4 \\
Pfeffer & 10 & 1 \\
EDG & 1 & 0 \\
\hline
\end{tabular}

A família optou por tratamento conservador e esse paciente veio a falecer com cinco meses de evolução.

\section{DISCUSSÃO}

Raramente, sintomas psiquiátricos são a única apresentação de um tumor cerebral. Qualquer tipo de sintoma psiquiátrico pode ocorrer com tumores cerebrais e muitas vezes simular demência. Infelizmente, os sintomas não têm, em geral, qualquer valor localizatório. Psicoses de início recente, sintomas de memória e humor, ocorrência de novos sintomas ou sintomas atípicos, como alterações da personalidade e anorexia sem sintomas dismórficos corporais, sugerem uma bateria de exames, incluindo neuroimagem¹. Outros transtornos neurológicos, como acidentes vasculares encefálicos, parkinsonismo primário, coreia de Huntington e epilepsias, também podem se revelar por meio de sintomas psiquiátricos, que, muitas vezes, surgem como primeira manifestação 22 .

Técnicas sofisticadas de neuroimagem, cada vez mais acessíveis, como a tomografia computadorizada (TC), a ressonância magnética (RM) e a tomografia por emissão de fóton único (SPECT), podem ser utilizadas como recurso complementar na prática psiquiátrica, em casos selecionados nos quais dados da história clínica, exame psíquico, físico e /ou neurológico trazem alguma suspeita da presença da condição médica geral concomitante ${ }^{23}$.

Neste caso, o exame de RM foi obrigatório pela suspeita de DA; os exames de imagem nos fornecem substrato para diferenciar as várias demências (vascular, lobar frontotemporal, Alzheimer, entre outras). Portanto, deve ser solicitada em todos os casos de suspeição dessas doenças. Apesar de ser um caso de evolução rápida, não pensamos em demências rapidamente progressivas de causa infecciosa porque, clinicamente, o paciente não apresentou febre nem manifestações sistêmicas de toxemia. No diagnóstico diferencial de Creutzfeldt-Jakob, soubemos que as manifestações eram de origem neurológica com vários sinais (ataxia de marcha, afasia, perda visual etc.) nas fases iniciais da doença e nosso paciente não apresentava sinais e sintomas neurológicos. 
Pensamos em DA pelo quadro clínico apresentado, mas o tempo curto de evolução (três meses) chamou a atenção para outra etiologia, sendo que na doença clássica seu início é insidioso, de um a dois anos, até que surjam as alterações comportamentais que não costumam se instalar nas fases iniciais da doença. Em nosso paciente, foi concomitante com as alterações de memória. A RM foi fundamental para trazer a etiologia tumoral como a causa dos sintomas neuropsiquiátricos do paciente e orientar a conduta.

A presença do edema cerebral é frequente no GBM e, pela agressividade desse tumor, em $60 \%$ deles há infiltração tumoral no edema. Apesar de a massa tumoral rechaçar cranialmente o hipocampo, o papel do edema cerebral também é importante nesse rechaço porque, com o uso da dexametasona, os sintomas do paciente melhoraram acentuadamente (memória e comportamento). A terapêutica desses tumores engloba duas vertentes: a de suporte e a definiti$\mathrm{va}^{24}$. O tratamento definitivo inclui a cirurgia, a radioterapia e a quimioterapia. A disseminação precoce de células malignas do GBM torna essa neoplasia incurável cirurgicamente. A cirurgia agressiva em que se remove a maior parte do tumor reduz o número de células cancerosas que necessitam de tratamento e, muitas vezes, remove o núcleo hipóxido do tumor, que é relativamente resistente à radiação e inacessível à quimioterapia ${ }^{25}$. O tratamento de suporte tem como objetivos o alívio sintomático e a melhoria da função neurológica. O agente de primeira linha são os corticosteroides (dexametasona). Anticonvulsivantes profiláticos não devem ser administrados em pacientes que não tiveram convulsão. É de salientar que, potencialmente, todos os gliomas malignos recidivarão, seja qual for o tratamento inicial realizado ${ }^{22}$. No caso, foi assumida uma conduta expectante em concordância com a família.

\section{CONCLUSÃO}

A apresentação atípica de sintomas psiquiátricos pode levar a uma variedade de erros diagnósticos. Causas orgânicas, incluindo tumores cerebrais, deveriam ser consideradas nessas circunstâncias. No caso apresentado, o diagnóstico foi de demência de Alzheimer com comportamento das demências rapidamente progressivas, que podem acontecer nos tumores malignos primários ou secundários. O tempo de doença era de três meses e havia comprometimento de memória e alterações comportamentais que se manifestaram com pouco intervalo de tempo. Os distúrbios de comportamento na DA clássica surgem após um a dois anos de doença, período que predominam os sintomas de memória. A RM foi solicitada para elucidação diagnóstica e é o exame que mostra particularidades dos vários tipos de demência, sendo necessária sua solicitação em casos de suspeita de demência. Em nosso paciente, a revelação do tumor orientou a conduta e justificou os achados clínicos. Após a introdução da dexametasona, o paciente apresentou regressão dos sintomas com repercussão nos testes neuropsicométricos em relação à pontuação antes da medicação.

\section{CONTRIBUIÇÕES INDIVIDUAIS}

José Eduardo Martinelli - Participou da elaboração do estudo, interpretação dos dados e revisão da versão final.

Juliana Francisca Cecato - Participou da coleta de dados, da aplicação dos testes neuropsicométricos e da revisão do estudo.

\section{CONFLITOS DE INTERESSE}

Os autores declaram não possuir conflitos de interesse.

\section{REFERÊNCIAS}

1. Madhusoodanan S, Ting MB, Ugur U. Psychiatric aspects of brain tumors: a review. World J Psychiatry. 2015;5(3):273-85.

2. Damasceno BP. Demências. In: Guariento MH, Neri AL (orgs.). Assistência Ambulatorial ao Idoso. Campinas: Alínea, 2010. p. 243-54.

3. Zajączkowska M, Kryszkowski W, Talarowska M, Gałecki P. Psychiatric symptoms as possible brain tumor complications: a case report. Med Sci Tech. 2015;56:73-7.

4. Kelley BJ, Boeve BF, Josephs KA. Rapidly progressive young-onset dementia. Cogn Behav Neurol. 2009;22(1):22-7.

5. Geschwind MD, Shu H, Miller BL. Rapidly progressive dementia. Ann Neurol. 2008;64(1):97108.

6. Madhusoodanan S, Patel S, Reinharth J, Hines A, Serper M. Meningioma and psychiatric symptoms: a case report and brief review. Ann Clin Psychiatry. 2015;27(2):126-33.

7. Engelhardt E. Demências rapidamente progressivas: uma revisão breve. Rev Bras Neurol. 2010;46(2):5-15.

8. Silva MC, Miotto EC, Aguiar PHP. Investigação neuropsicológica pré-operatória em pacientes com glioma de baixo grau. J Bras Neurocirurg. 2007;18(3):35-9.

9. Lishman WA. Organic psychiatry. The psychological consequences of cerebral disorder. 3rd ed. Offord: Blackwell Science, 2005.

10. Silva MC, Aguiar PHP. Gliomas e qualidade de vida. J Bras Neurocir. 2006;17(2):61-6.

11. Geschwind MD, Shu H, Haman A, Sejvar JJ, Miller BL. Rapidly Progressive Dementia. Ann Neurol. 2008;64(1):97-108.

12. Geschwind MD, Haman A, Miller BL. Rapidly Progressive Dementia. Neurol Clin. 2007;25:783-807.

13. American Psychiatric Association. Diagnostic and Statistical Manual of Mental Disorders DSM-5 (5th ed.). Washington, DC. 2014.

14. Petersen RC, Stevens J, Ganguli M, Tangalos EG, Cummings J, DeKosky ST. Pratice parameter: early detection of dementia: mild cognitive impairment. Neurology. 2001;56:1133-42.

15. Alvares JL, Ortiz LFA. Nuevos criterios disgnósticos de la demência y la enfermidade de Alzheimer: una vision desde la psicogeriatria. Psicogeriatria. 2015;5(1):3-14.

16. Folstein MF, Folstein SE, McHugh PR. Mini-Mental State: a practical method for grading the cognitive state of patients for the clinician. J Psychiatr Res. 1975;12:189-98.

17. Roth M, Tym E, Mountjoy CQ, Huppert FA, Hendrie H, Verma S, et al. CAMDEX. A standardised instrument for the diagnosis of mental disorder in the elderly with special reference to the early detection of dementia. Br J Psychiatry. 1986;149:698-709. 
18. Pfeffer Rl, Kurosaki TT, Harrah CH Jr, Chance JM, Filos S. Measurement of functional activities in older adults in the community. J Gerontol. 1982;37:323-9.

19. Mendez MF, Ala T, Underwood K. Development of scoring criteria for the clock drawing task in Alzheimer's disease. J Am Geriatr Soc. 1992;40:1095-9.

20. Shulman KI, Gold DP, Cohen CA, Zucchero CA. Clock-drawing and dementia in the community: a longitudinal study. Int J Geriatr Psychiatry. 1993;8(6):487-96.

21. Yesavage JA, Brink TL, Rose TL, Lum 0, Huang V, Adey M, et al. Development and validation of a geriatric depression screening scale: a preliminary report. J Psychiatr Res. 1983;17(1):37-49.
22. Derouesné C. Brain tumors, cognitive and mental disorders in adults. Geriatr Psychol Neuropsychiatr Vieil. 2015;13(2):187-94.

23. Skaf CR, Yamada A, Akamine S, Busatto Filho G. Meningeoma frontal numa paciente com síndrome depressiva maior crônica. Rev Bras Psiquiatr. 1999;21:114-6.

24. De Angelis LM, Loeffler JS, Mamelak NA. Primary brain tumors. Cancer managment. A multidisciplinary approach. 8th edition. 2004;28:591-606.

25. Correia J, Alexandre JC, Dias C, Matos LC, Martins I, Ribeiro P, et al. Glioblastoma multiforme. A propósito de um caso clínico. Casos clínicos. Med Interna. 2009;16(1): 27-32. 